\title{
Evaluación del estado nutricional en niños con parálisis cerebral infantil
}

\author{
Evaluation of nutritional status in children with cerebral palsy
}

\author{
Laura Godoy Sánchez', Juan Fernando Ojeda', Mirta Mesquita'
}

\begin{abstract}
RESUMEN
Introducción: La desnutrición en pacientes con Parálisis cerebral infantil (PC) aumenta la morbimortalidad, generando alta demanda económica al estado y la familia. Objetivo: Describir estado nutricional de niños con PC. Materiales y Métodos: Estudio observacional, descriptivo con componente analítico, prospectivo. Fueron incluidos niños de 0-18 años con PC que acudieron al Departamento de Neurología en setiembre y octubre del 2017. Variables: edad, sexo, antecedentes perinatales de Riesgo, antecedentes de infección del sistema nervioso central, estado nutricional, talla, perímetro cefálico, grado de discapacidad motora, vía de alimentación, trastornos relacionados a la alimentación, síntomas asociados a reflujo gastroesofágico. Los datos fueron analizados en SPSSv21, utilizando estadística descriptiva e inferencial. Protocolo aprobado por el Comité de Investigación y Ética institucional, con consentimiento informado. Resultados: se incluyeron 42 pacientes. La mediana de edad fue 5 años. Predominio de sexo femenino: $52,4 \%$ (22/42). El $83,3 \%(35 / 42)$ tenía antecedentes perinatales de riesgo (80\% encefalopatía hipóxico-isquémica); antecedentes de infección del sistema nervioso central 9.5\%(4/42); se encontró desnutrición en 35,7\% (15/42); alteración de talla $76,2 \%$; todos los lactantes tenían alterado el perímetro cefálico; discapacidad motora Grado 4 en $61,9 \%(26 / 42)$; se alimentaban por vía oral: 78,6\%(36/42); tenían trastornos de alimentación, 36/42; síntomas asociados a reflujo gastroesofágico 69\%; se relacionó desnutrición con grado de discapacidad y se encontró diferencia significativa, sobretodo con Grado 4 ( $\mathrm{p}<0,005)$; en relación al grado de discapacidad, trastornos de alimentación y síntomas de reflujo gastroesofágico, se encontró diferencia significativa con mayores grados de discapacidad. Conclusiones: La frecuencia de desnutrición fue 35,7\%. El estado nutricional se relacionó con el grado de discapacidad motora.
\end{abstract}

Palabras claves: Parálisis infantil, estado nutricional

\section{ABSTRACT}

Introduction: Malnutrition in patients with infantile cerebral palsy (CP) increases morbidity and mortality, generating a high economic burden on he state and the family. Objective: To describe the nutritional status of children with CP. Materials and Methods: This was an observational, descriptive study with analytical and prospective components. Children aged 0-18 years with $\mathrm{CP}$, who consulted at the Department of Neurology in September and October 2017, were included. Variables: age, sex, perinatal risk history, history of central nervous system infection, nutritional status, height, head circumference, degree of motor disability, food intake route, eating-related disorders, and symptoms associated with gastroesophageal reflux. The data were analyzed in SPSSv21, using descriptive and inferential statistics. The protocol was approved by the Institutional Research and Ethics Committee, and we obtained informed consent. Results: 42 patients were included. The median age was 5 years, the subjects were predominantly female: $52.4 \%$ (22/42). 83.3\% (35/42) had a perinatal risk history, $(80 \%$ hypoxic-ischemic encephalopathy); a history of central nervous system infection was noted in 9.5\% (4/42); malnutrition was found in $35.7 \%$ (15/42); height alteration was found in $76.2 \%$; all infants had alterations in their head circumference measurements; motor impairment Grade 4 was noted in $61.9 \%$ (26/42); oral feeding was noted in $78.6 \%$ (36/42); 36/42 had eating-related disorders; $69 \%$ had symptoms associated with gastroesophageal reflux; malnutrition was related to degree of disability and a significant difference was found, especially with Grade 4 ( $p<0.005)$; regarding degree of disability, eating-related disorders and gastroesophageal reflux symptoms, we found significant associations with higher degrees of disability. Conclusions: The frequency of malnutrition was $35.7 \%$. The nutritional status was related to the degree of motor disability.

Keywords: Infantile paralysis, nutritional status

\footnotetext{
${ }^{1}$ Hospital Pediátrico Niños de Acosta Nu. San Lorenzo, Paraguay.

Correspondencia: Laura Godoy Correo: legodoys@gmail.com

Conflicto de interés: Los autores declaran no poseer conflicto de interés

Recibido: 25/02/2019. Aceptado: 10/03/2019

DOI:https://doi.org/10.31698/ped.46012019004
}

20 Pediatr. (Asunción). 2019; 46(1):20-25 (enero - abril) 


\section{INTRODUCCIÓN}

La parálisis cerebral (PC) es la causa más frecuente de discapacidad en la población infantil. Este término describe el síndrome producido por daño del sistema nervioso central (SNC) durante períodos críticos del desarrollo, con manifestaciones clínicas variables, que comparten el compromiso neurológico motor $^{(1,2)}$.

En países desarrollados, la incidencia de PC es 1,5-3 por 1000 recién nacidos ${ }^{(1,3)}$.

La malnutrición y las alteraciones neurológicas constituyen problemas de salud frecuentemente superpuestos. Son varios los motivos por los cuales los niños con PC tienen problemas para la alimentación. Entre los reportados en diversos estudios se citan los trastornos asociados al compromiso motor, que impiden que el niño pueda comer y beber adecuadamente, con pobre ingesta calórica, ocasionando desnutrición y daño adicional al $\mathrm{SNC}^{(1,4)}$.

En los países de bajos ingresos, ante la ausencia de centros multidisciplinarios adecuados para estos niños, es la familia, y, principalmente la madre la responsable del bienestar del niño. De modo que el bienestar del niño depende en gran medida de la salud emocional de la madre o cuidadora principal. La calidad de vida de los cuidadores de estos niños también se ha asociado a dificultades en la alimentación, asi como la presencia de reflujo gastroesofágico y disfunción orofaríngea, muy frecuentemente observado en niños con $\mathrm{PC}^{(5,6)}$.

Como consecuencia de las dificultades para recibir suficiente aporte calóricos, los niños con PC frecuentemente presentan diferentes grados de desnutrición. En países de bajos ingresos de Asia y África se han reportado prevalencias de desnutrición en niños con PC d de 57 a $86 \%$ respectivamente ${ }^{(7,8)}$.

La desnutrición en estos pacientes aumenta la morbimortalidad, días cama en hospitales, generando alta demanda económica al estado y familia. Una intervención adecuada mejora su calidad de vida ${ }^{(4,9)}$. Hasta donde se investigo no se encontró reportes sobre la prevalencia de PC así como tampoco sobre el estado nutricional de niños con PC en Paraguay. El objetivo de este trabajo fue describir el estado nutricional en niños con PC que acuden al departamento de neurología de un hospital pediátrico de referencia del país.

\section{MATERIALES Y MÉTODOS}

Estudio observacional, descriptivo con componente analítico, prospectivo. Se incluyeron pacientes de $0 \mathrm{a}$ 18 años que acudieron al departamento de neuropediatria con diagnóstico de PC, de setiembre a octubre del 2017.

Instrumentos de medición: En los niños menores de 2 años para la medición del peso, se utilizó la balanza pediátrica (marca Balmark) y en los niños con edad $\geq 2$ años, balanza digital de marca Tokio. En aquellos niños que no podían ponerse de pie se realizó un peso indirecto de dos pasos; el primero pesando a la madre sola y el segundo con el hijo en brazos, restándole el peso materno se obtenía el del niño.

La talla en los menores de 2 años fue tomada con el tallímetro de lactantes, y en los mayores de 2 años, con el tallímetro de pie o una cinta métrica inextensible, sobre una superficie lisa.

En los pacientes con espasticidad severa, deformidades esqueléticas o incapacidad de mantenerse de pie se obtuvo la longitud de la tibia (LT) del miembro inferior derecho colocando en posición sentada o acostada y con una pierna cruzada horizontalmente sobre la otra. Se utilizó una cinta métrica para medir la distancia comprendida entre el cóndilo medial de la tibia y el borde inferior del maléolo interno, una vez obtenido el dato se ingresó el valor en la fórmula establecida $(3,26 \times \mathrm{LT})+30,8 \pm$ 1,4 (38). Con los datos antropométricos obtenidos, el estado nutricional se estableció de acuerdo a las tablas de crecimiento de la OMS para niños con PC.

Las variables estudiadas fueron edad, sexo, antecedentes perinatales de Riesgo, antecedentes de infección del sistema nervioso central, estado nutricional, talla, perímetro cefálico, grado de discapacidad motora, vía de alimentación, trastornos relacionados a la alimentación, síntomas 
asociados a reflujo gastroesofágico.

El cálculo del tamaño muestral estableció que, para conseguir una precisión del $5 \%$ en la estimación de una proporción, mediante un intervalo de confianza del 95\%, fue necesario incluir 42 participantes. Los datos fueron analizados en el sistema SPSSv21. Las variables cualitativas se expresaron en porcentajes y las cuantitativas en medianas. Para la asociación de variables se utilizó la prueba de Chi cuadrado. Se consideró un error alfa inferior a $5 \%$.

El protocolo fue aprobado por el Comité institucional de Investigación y Ética de la investigación.

\section{RESULTADOS}

Durante el periodo de estudio fueron incluidos 42 pacientes. En cuanto a los datos demográficos, la mediana de edad fue 5 años con un mínimo de 1 y un máximo de 16 años (intervalos intercuartilicos: P25 2 años, P75 8 años). Se encontró un leve predominio de sexo femenino: $52,4 \%(22 / 42)$.

El 83,3\% (35/42) de los pacientes con PCI tenían antecedentes perinatales de riesgo. los cuales se citan en la Tabla 1. El 9.5\% (4/42), tenían de antecedentes de infección del sistema nervioso central y todos fueron meningitis bacteriana aguda. Un paciente tenía como antecedente, traumatismo craneano producido a los 8 meses de vida.

El estado nutricional en relación al peso y la talla se aprecia en la Tabla 2.

En los lactantes (7/42), el 100\% presentó alteración del perímetro cefálico, constatándose que $5 / 7$ tenían microcefalia, $1 / 7$ riesgo de microcefalia y $1 / 7$ macrocefalia.

En la Tabla 3 se observan el grado de discapacidad motora que presentaron los pacientes.

La mayoría de los pacientes presentaban trastornos de la alimentación, 36/42 (85.7\%), como se detalla en la Tabla 4. Muchos pacientes presentaban más de una dificultad para la alimentación.
Tabla 1. Tipos de antecedentes perinatales de riesgo en pacientes con parálisis cerebral que acuden al Departamento de Neurología $\mathrm{n}=35$.

\begin{tabular}{lcc}
\hline Tipo de Antecedente perinatal de riesgo & N & \% \\
\hline Encefalopatía hipoxico-isquémica & 28 & 80 \\
Microcefalia & 3 & 8.5 \\
Encefalopatía hiperbilirrubinémica & 2 & 5.7 \\
Infecciones connatales & 2 & 5.7 \\
Total & $\mathbf{3 5}$ & $\mathbf{1 0 0}$ \\
\hline
\end{tabular}

Tabla 2. Estado nutricional y de la tala de pacientes con diagnóstico de Parálisis cerebral infantil que acuden al Departamento de Neurología. N=42

\begin{tabular}{|c|c|c|c|c|c|c|c|c|}
\hline & \multicolumn{2}{|c|}{ Adecuado } & \multicolumn{2}{|c|}{ Riesgo de Desnutrición } & \multicolumn{2}{|c|}{ Desnutrición } & \multicolumn{2}{|c|}{ Sobrepeso } \\
\hline & $\mathrm{N}$ & $\%$ & $\mathrm{~N}$ & $\%$ & $\mathrm{~N}$ & $\%$ & $\mathrm{~N}$ & $\%$ \\
\hline Estado & 20 & 46.7 & 5 & 11.9 & 15 & 35.7 & 2 & 4.8 \\
\hline \multicolumn{9}{|l|}{ nutricional } \\
\hline & \multicolumn{2}{|c|}{ Adecuado } & \multicolumn{2}{|c|}{ Riesgo de Talla Baja } & \multicolumn{2}{|c|}{ Talla Baja } & \multicolumn{2}{|c|}{ Talla muy Baja } \\
\hline & $\mathrm{N}$ & $\%$ & $\mathrm{~N}$ & $\%$ & $\mathrm{~N}$ & $\%$ & $\mathrm{~N}$ & $\%$ \\
\hline Talla/Edad & 10 & 23.8 & 12 & 28.6 & 6 & 14.3 & 14 & 33.3 \\
\hline
\end{tabular}

Tabla 3. Grado de discapacidad motora gruesa en pacientes con parálisis cerebral que acuden al Departamento de Neurología. $\mathrm{N}=42$

\begin{tabular}{lcc}
\hline Grado de discapacidad & N & \% \\
\hline Grado 4 & 26 & 61.9 \\
Grado 5 & 7 & 16.7 \\
Grado 2 & 5 & 11.9 \\
Grado 3 & 4 & 9.5 \\
Total & $\mathbf{4 2}$ & $\mathbf{1 0 0}$ \\
\hline
\end{tabular}

Tabla 4. Trastornos de alimentación de los pacientes con Parálisis Cerebral Infantil que acuden al Departamento de Neurología.

\begin{tabular}{lc}
\hline Trastornos de alimentación & N \\
\hline Dificultad para la deglución & 22 \\
Dificultad para la succión & 21 \\
\hline Rechazo a la comida & 15 \\
Regurgitación y/o vómitos postprandiales & 10 \\
\hline Falta de cierre de labios alrededor de la cuchara & 9 \\
\hline Apertura disminuida de la boca & 3 \\
\hline
\end{tabular}

En cuanto a la forma de alimentación, se consideraron las siguientes vías: oral, por sonda y por gastrostomía, tal como se observa en la Figura 1.

Se observaron síntomas asociados a reflujo gastroesofágico en el 69\% (29/42), de los niños los cuales se describen en la Tabla 5.

Se analizó la presencia de desnutrición en relación al grado de discapacidad y se encontró una diferencia 
significativa sobretodo en el grado 4. Ver Tabla 6.

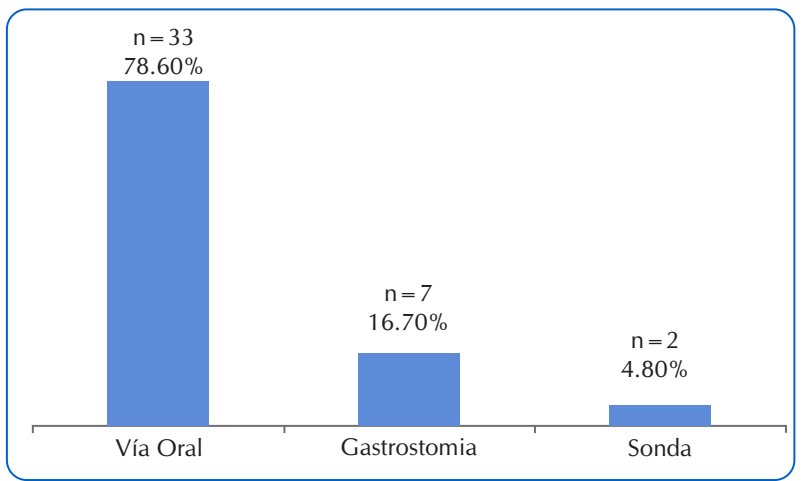

Figura 1. Forma de alimentación de los pacientes con parálisis cerebral que acuden al Departamento de Neurología. $\mathrm{N}=42$

Tabla 5. Síntomas asociados a reflujo gastroesofágico en pacientes con parálisis cerebral que acuden al Departamento de Neurología. $\mathrm{n}=29$

\begin{tabular}{lcc}
\hline Síntomas asociados a reflujo gastroesofágico & N & $\%$ \\
\hline Infecciones respiratorias recurrentes & 26 & 89.7 \\
Sibilancias & 26 & 89.7 \\
Regurgitación y/o vomito postprandial & 10 & 34.5 \\
Tos crónica & 21 & 72.4 \\
Tos durante o después de la alimentación & 22 & 75.9 \\
Distonía extensora durante la alimentación & 6 & 20.7 \\
Estridor recurrente & 3 & 6.9 \\
Llanto durante o después de la alimentación & 4 & 13.8 \\
\hline
\end{tabular}

Tabla 6. Estado nutricional según grado de discapacidad motora en pacientes con parálisis cerebral que acuden al Departamento de Neurología. $\mathrm{N}=42$

\begin{tabular}{|c|c|c|c|c|c|}
\hline $\begin{array}{l}\text { Grado de } \\
\text { discapacidad }\end{array}$ & Adecuado & $\begin{array}{c}\text { Riesgo de } \\
\text { desnutrición }\end{array}$ & Desnutrición & Sobrepeso & p \\
\hline & $\mathrm{N}(\%)$ & $\mathrm{N}(\%)$ & $\mathrm{N}(\%)$ & $\mathrm{N}(\%)$ & \\
\hline $\begin{array}{l}\text { Grado } 2 \\
n=4\end{array}$ & $4(80)$ & 0 & 0 & 0 & \\
\hline $\begin{array}{l}\text { Grado } 3 \\
n=4\end{array}$ & $2(50)$ & $1(25)$ & 0 & $1(25)$ & $\begin{array}{c}0.007 \\
(*)\end{array}$ \\
\hline $\begin{array}{l}\text { Grado } 4 \\
n=26\end{array}$ & $8(30.8)$ & $3(11.5)$ & $15(57.7)$ & 0 & \\
\hline $\begin{array}{l}\text { Grado } 5 \\
\mathrm{n}=7\end{array}$ & $6(85.7)$ & $1(14.3)$ & 0 & 0 & \\
\hline
\end{tabular}

Con respecto al grado de discapacidad y la presencia de trastornos de alimentación, se encontró una diferencia significativa con mayores grados de discapacidad al igual que con la presencia de síntomas de reflujo gastroesofágico, que se observó en $2 / 4$ con discapacidad grado 3 , en 20/26 con grado 4 y en $7 / 7$ de los que presentaban grado $5(p<0,001)$.

\section{DISCUSIÓN}

En este trabajo se encontró que, más de la mitad de la población estudiada tenía estado nutricional alterado, de los cuales la gran mayoría se encontraban con diferentes grados de desnutrición. Cifras similares a un estudio realizado en Lima en el 2004 por Del Águila y col con cifras de 81,1\% de desnutrición en pacientes con $\mathrm{PC}^{(1)}$. Sin embargo son superiores a otro trabajo hecho en Brasil, publicado en el 2017 por Caselli y colaboradores que encontró $22 \%$ de desnutricion utilizando las curvas adaptadas para este grupo de niños ${ }^{(2)}$. En la población estudiada la mitad eran preescolares de sexo femenino, esta ligera predominancia de sexo difiere con un estudio realizado en el año 2011 por la Agencia de Cooperación Internacional de Japón (JICA) en tres departamentos de la Región Oriental del Paraguay que refiere que la discapacidad en menores de 15 años fue mayor en varones ${ }^{(10)}$.

Un alto porcentaje de la población en este estudio, presentó antecedentes perinatales de riesgo. En la mitad de ellos el diagnóstico se de PC se realizó hacia los 3 meses de edad y la gran mayoría tuvieron el diagnóstico de encefalopatía hipóxico isquémica como antecedente. Estos datos se corresponden a los reportes a nivel país que indican que, entre las causas de mortalidad neonatal, las lesiones debidas al parto ocupan el primer lugar y aún hasta el año de edad, estas lesiones son causa importante de discapacidad $^{(10)}$. La asociación entre PC y factores perinatales de riesgo se encontró en varios estudios. Una revisión sistemática de los factores de riesgo de PC en países desarrollados encontró como significativas a 10 entidades perinatales que son: anormalidades de la placenta, malformaciones congénitas, bajo peso de nacimiento, cesáreas de urgencias, asfixia perinatal, síndrome de aspiración meconial, hipoglicemia, síndrome de distress respiratorio, hipoglicemia y las infecciones neonatales ${ }^{(11)}$.

En cuanto a las malformaciones congénitas se encontró en el presente reporte con una frecuencia del 8,5\% que correspondió a microcefalia, por encima del porcentaje de malformaciones congénitas reportadas a nivel país, que es del 3 al $3,5 \%$. Esta diferencia podría deberse al azar, 
considerando el escaso número de la población ${ }^{(10,12)}$. Se observó también infecciones perinatales en dos casos atribuidos a toxoplasmosis y citomegalovirus, ambas relacionadas a secuelas severas del SNC. En poblaciones menos desarrolladas y de cultura islámica el parto domiciliario y la consanguinidad de los padres son factores de riesgo de $\mathrm{PC}^{(12)}$.

Un reporte australiano publicado en 2014 por Benfer y col, comparando la severidad de la discapacidad motora en dos poblaciones, una desarrollada y la otra con escaso desarrollo, demostró que los patrones son diferentes, siendo más severos en esta última población, indicando la importancia del manejo perinatal del parto y el entrenamiento de los médicos para el diagnóstico temprano e inicio de las terapias correspondientes ${ }^{(11)}$.En esta serie se encontró que la gran mayoría de los pacientes presentaron un grado elevado de discapacidad motora y que existía relación con antecedentes perinatales de riesgo.

La alteración del estado nutricional, particularmente la desnutrición es muy común en los pacientes con PCI, aun en países desarrollados ${ }^{(11)}$. Aunque no existen datos a nivel población, algunos reportes de países poco desarrollados reportan que más del $50 \%$ se encuentran desnutridos ${ }^{(13)}$. La lesión neurológica puede alterar en mayor o menor grado la función neuromuscular, lo cual ocasiona incoordinación en

\section{REFERENCIAS BIBLIOGRÁFICAS}

1. Del Águila A, Áibar P. Características nutricionales de niños con parálisis cerebral: ARIE - Villa El Salvador, 2004. An. Fac. med. [Internet]. 2006; [citado 26 de agosto de 2017]; 67(2):108-119. Disponible en: http://www.scielo.org.pe/ scielo.php?script=sci_arttext\&pid=S102555832006000200003\&lng=es.

2. Casseli TB, Lomazi EA, Montenegro MAS, BellomoBrandao MA, Assessment of nutritional status of children and adolescents with Spastic L Cerebral Palsy. Arq Gastroenterol.2017;45(3): 101-105.

3. Rosenbaum P. Cerebral palsy: what parents and doctors want to know?. A clinical review. British Medical Journal. 2003;326:970-4. los procesos de succión, masticación, deglución y digestión, y a su vez, lleva a una ingesta insuficiente y malnutrición en un porcentaje variable ${ }^{(12)}$. En el presente estudio se encontró que el estado nutricional se asocio al grado de discapacidad. Los tipos de trastornos encontrados son similares a los reportados por otros autores ${ }^{(1.15,16)}$.

Los trastornos en la alimentación se presentaban en la mayoría de los niños, siendo los más frecuentes: dificultad para la masticación, incoordinación motora o falla de control cefálico, sialorrea, dificultad para la deglución, tal como se describe en varios estudios $^{(14.15,16)}$.

En cuanto a la forma de alimentación, se encontró que la mayoría lo hacía por vía oral y en un menor número de casos por gastrostomía y sonda nasogástrica. Los niños con gastrostomía, tenían mejor estado nutricional. Las complicaciones más frecuentemente asociadas al RGE fueron las respiratorias.

\section{CONCLUSIONES}

La gran mayoría de los pacientes con PCI tenían malnutrición con predominio de déficit, desnutrición $(35,7 \%)$ y riesgo de desnutrición, asociado a la discapacidad motora.
4. Le Roy OC, Reballo GMJ, Moraga F, Díaz X, Castillo Duran C. Nutrición del niño con enfermedades neurológicas prevalentes. Revista Chilena de Pediatría 2010; 81(2):103-113.

5. Polack S, Adams M, O' Banion D, Baltussen M, Asante S, Kerac M. et al. Children with cerebral palsy in Ghana: malnutrition, feeding challenges, and caregiver quality of life. Dev Med Child Neurol. 2018; 60(9):914-921.

6. Asgarshirazi M, Farokhzadeh-Soltani M, Keihanidost Z, Shariat M. Evaluation of Feeding Disorders Including Gastro-Esophage Reflux and Oropharyngeal Dysfunction in Children With Cerebral Palsy.Family Reprod Health. 2017; 11(4):197-20. 
7. Aydin K, Turkish Cerebral Palsy Study Group. A multicenter cross-sectional study to evaluate the clinical characteristics and nutritional status of children with cerebral palsy. Clin Nutr ESPEN. 2018; 26:27-34.

8. Adamu AS, Sabo UA, Gwarzo GD, Belonwu RO. Nutritional status in cerebral palsy: A Cross-sectional comparative survey of children in Kano, Nigeria. Niger Postgrad Med J. 2018; 25(3):156-160.

9. García Zapata LF, Restrepo Mesa SL. Alimentar y nutrir a un niño con parálisis cerebral. Una mirada desde las percepciones. Investigación y Educación en Enfermería. 2011;29(1):28-395.

10. UNICEF. Situación del derecho a la salud materna, infantil y adolescente en Paraguay [Internet]. Asunción: UNICEF; 2013. [Citado 20 de agosto de 2017]. Disponible en: https://www.unicef.org/paraguay/spanish/pyinformesalud.pdf

11. Benfer K, Jordan R, Bandaranayke S, Finn C, Ware R, Boyd R. Motor Severity in Children With Cerebral Palsy Studied in a High-Resource and Low-Resource Country. Pediatrics. 2014; 134(6):1594-602.
12. Bangash AS, Hanafi MZ, Idrees R. Risk factors and types of cerebral palsy. The Journal of the Pakistan Medical Association. 2014;64(1):103-107.

13. Brooks J, Día S, R Shavelle, Strauss D. Bajo peso, la morbilidad y la mortalidad en niños con parálisis cerebral: nuevas tablas de crecimiento clínicos. Pediatrics. 2011; 128(2):299-307.

14. Karagiozoglou-Lampoudi T, Daskalou E, Vargiami E, Zafeiriou D. Identification of feeding risk factors for impaired nutrition status in paediatric patients with cerebral palsy. Acta Paediatr. 2012; 101(6):649-654.

15. Bacco JL, Araya F, Flores E, Peña N. Trastornos de la alimentación y deglución en niños y jóvenes portadores de parálisis cerebral: abordaje multidisciplinario. Revista Médica Clínica Las Condes. 2014; 25(2):176-393.

16. Barrón F, Riquelme M, Elizondo J, Químbar A. Reflujo gastroesofágico y problemas respiratorios en parálisis cerebral infantil. [Internet] México: Instituto Nuevo Amanecer; 2008 [citado junio de 2017]. Disponible en: http://www.nuevoamanecer.edu.mx/imgs/pdf/ReflujoGa stroesofagicoProblRespiratorios.pdf 